\title{
La tuberculose dans le Nouveau Monde : Christophe Colomb n'avait peut-être pas tous les torts
}

Reconstruire l'histoire de maladies infectieuses telles que la tuberculose, le paludisme ou le SIDA peut être riche d'enseignement pour la compréhension de ces maladies ainsi que pour envisager des politiques d'éradication rationnelles.

Notre source d'information sur les maladies dans les anciennes populations repose essentiellement sur des constatations archéologiques portant sur les corps eux-mêmes. Pour les maladies infectieuses, les lésions observées sur les os ou les tissus mous sont rarement pathognomoniques. Aussi est-on tributaire de l'identification de l'agent infectieux directement à partir des restes humains. Malheureusement, les méthodes classiques d'isolement par culture sont inefficaces dans les tissus archéologiques. Une approche alternative repose sur la mise en évidence de l'ADN de l'agent pathogène qui aurait persisté dans la lésion. Une équipe américaine vient de valider cette approche en isolant une séquence spécifique d'ADN de Mycobacterium tuberculosis à partir d'un poumon provenant d'une femme péruvienne âgée de 1000 ans spontanément momifiée [1].

La tuberculose a été choisie pour tester la faisabilité de cette approche car la présence de cette maladie dans le Nouveau Monde avant l'arrivée de Christophe Colomb est sujette à controverses et la nature des lésions observées sur les corps est ambiguë. La cyphose, due à la déformation spinale (Mal de Pott) secondaire à l'atteinte tuberculeuse du rachis, qui a servi à identifier la tuberculose dans le Nouveau Monde avant 1492, peut aussi être causée par une variété de micro-organismes, dont le champignon Coccidioides immitis. La présence d'un bacille acido-alcoolo résistant dans un abcès paravertébral chez un enfant péruvien vieux de 800 ans rend le diagnostic de tuberculose pré-colombienne plus vraisemblable. Toutefois, toutes les espèces de Mycobactéries (et même celles du genre Nocardia) peuvent être acidoalcoolo résistantes. De plus, de nombreuses espèces de mycobactéries vivent dans le sol et il a été récemment prouvé qu'elles pouvaient coloniser des tissus humains enterrés. Les chercheurs ont examiné un ganglion hilaire et une lésion pulmonaire nodulaire d'une femme de 40 45 ans. Le corps a été exhumé d'un site mortuaire utilisé par les Chiribaya, une population d'agriculteurs qui occupait une région côtière au sud du Pérou depuis 10001300 avant Jésus-Christ. Seuls les poumons montraient des signes macroscopiques et histologiques en faveur de la tuberculose chez cette femme dont un prélèvement hépatique a été daté à $1040 \pm 44$ ans par la méthode au ${ }^{14} \mathrm{C}$, alors que des lésions osseuses évocatrices de tuberculose avaient été retrouvées sur d'autres corps du site mortuaire. Aucun bacille acido-alcoolo résistant n'a toutefois été vu dans les lésions pulmonaires.

Un fragment spécifique de l'ADN de M. tuberculosis a été amplifié par PCR (polymerase chain reaction) en utilisant une méthode de "PCR emboîtée" (nested PCR) où le premier produit de PCR (123 pb) est réamplifié avec un jeu d'amorces oligonucléotidiques internes donnant un frag. ment de $97 \mathrm{pb}$. Ce fragment fait partie d'une séquence répétitive d'insertion (IS6110) de 1361 pb qui existe en 10-16 copies par cellule et dont la présence a été fortement corrélée à une tuberculose clinique. Les auteurs affirment avoir pris des mesures très strictes pour lutter contre les contaminations. Le ganglion s'est avéré positif par PCR et le séquençage des produits d'amplification a montré que la séquence obtenue était identique à la séquence IS6110 des M. tuberculosis actuelles, séquence extrêmement conservée parmi les souches isolées jusqu'à ce jour. Ainsi donc, grâce à ce travail, la présence de tuberculose humaine sur le continent américain avant l'arrivée de Christophe Colomb a pu être confirmée.

E.D.

1. Salo WL, Aufderheide AC, Buikstra J Holcomb TA. Identification of Mycobacterium tuberculosis DNA in a pre-Columbian Peruvian mummy. Proc Natl Acad Sci USA 1994 ; 91 : 2091-4. 